\title{
La modelación en la producción de conocimiento matemático: el caso de la función seno ${ }^{1}$
}

\author{
The modeling in the production of mathematical \\ knowledge: the case of the sine function \\ A modelagem na produção do conhecimento \\ matemático: o caso de a função seno
}

Recibido: mayo 2013

Aceptado: agosto 2013
Juan Fernando Molina Toro ${ }^{2}$

Jhony Alexander Villa-Ochoa ${ }^{3}$

\section{Resumen}

En el documento se presentan los resultados parciales de una investigación en la que se propuso un proceso de modelación matemática para estudiar algunos aspectos de la función trigonométrica seno. Se fundamenta la pertinencia de la modelación como una manera de desarrollar otro tipo de representaciones (modelos) matemáticas para el trabajo particular con conceptos, que permiten establecer relaciones entre variables matemáticas presentadas en algunos problemas del entorno, y exigen la elaboración de un modelo para su acertada consecución. Finalmente se presenta un diseño metodológico para trabajar un proceso de modelación con simulaciones, desde las cuales emergen conceptos matemáticos subyacentes a las funciones trigonométricas seno y coseno.

Palabras clave: Trigonometría; aprendizaje; procesos cognitivos; modelación matemática; simulación; trigonometría.

\begin{abstract}
The paper presents the partial results of an investigation in which we proposed a mathematical modeling process for the study some aspects of the trigonometric sine function. It argues for the relevance of modeling as a way to develop other kinds of representations (models) for the particular job math concepts, that establish mathematical relationships between variables presented in some environmental problems, and require the development of a model for successful achievement. Finally, a design methodology for working with process modeling simulations, from which emerge mathematical concepts underlying the trigonometric functions sine and cosine.
\end{abstract}

Keywords: Trigonometry; learning cognitive processes, mathematical modeling, simulation, trigonometry.

1 Artículo de Investigación

2 Universidad de Medellín. Contacto: juanfdomolina@gmail.com

3 Universidad de Antioquia. Contacto: jhonyvilla@gmail.com. 


\section{Resumo}

O artigo apresenta os resultados parciais de uma investigação na qual foi proposto um processo de modelagem matemática para o estudo de alguns aspectos da função seno trigonométrico. Ele defende a relevância da modelagem como uma forma de desenvolver outros tipos de representações (modelos) para os conceitos de matemática trabalho específico, que estabelecem relações matemáticas entre as variáveis apresentadas em alguns problemas ambientais, e exigem o desenvolvimento de um modelo para realização bem sucedida. Finalmente, uma metodologia de projeto para trabalhar com simulações de modelagem de processos, a partir do qual emergem conceitos matemáticos subjacentes a funções trigonométricas seno e cosseno.

Palavras-chave: Trigonometria; aprendizagem processos cognitivos, modelagem matemática, simulação, trigonometria.

\section{Presentación del problema}

Durante varias décadas el estudio de la trigonometría ha estado inmerso en los currículos escolares de las instituciones educativas colombianas; su importancia y trascendencia han permitido que la estructura temática sea insumo, entre otras; para el estudio de la física o la geometría analítica. La trigonometría supone elementos que facilitan las relaciones entre magnitudes y permiten la interpretación de algunos fenómenos periódicos como el estudio de las ondas y las vibraciones. Sin embargo, hay una serie de dificultades que también han permanecido latentes en las aulas de clase cuando se aborda el estudio de la trigonometría; algunas reflejan conflictos que anteceden a la formación de conceptos y habilidades en el estudio de la matemática, impedimentos para establecer relaciones entre cantidades (el doble de, la mitad de, la tercera parte de...) y otras tienen que ver con manejo del lenguaje simbólico y su decodificación (Mora, Nieto, Polanía, Romero, y González, 2012).

Al parecer el excesivo énfasis en la aplicación de procedimientos ha dejado de lado la reflexión y el análisis de otras situaciones que van más ligadas al contexto en el cual se desarrolla la trigonometría (relaciones métricas en el triángulo rectángulo, solución de situaciones problema, entre otras), y dejan frecuentemente todo el trabajo de aula con las razones trigonométricas dirigido a la repetición o a la aplicación de algorítmos. Es así como ha perdido sentido "resolver problemas" en los cuales se aprecie la naturaleza y la importancia de la trigonometría.

En Colombia, los programas para la enseñanza de la matemática deben seguir las orientaciones emitidas desde el Ministerio de Educación Nacional, en este caso, el texto de Estándares Básicos de Competencias en Matemáticas declaran como un propósito al finalizar el ciclo de Educación Media, que los estudiantes estén en capacidad de "describir y modelar fenómenos periódicos del mundo real usando relaciones y funciones trigonométricas" ( $\mathrm{p}$. 88). Esta meta, en esencia, expresa dos características particulares que se abordaron implícitamente dentro de la investigación las cuales se mencionará más adelante, la descripción en cuanto a la posibilidad de explicar y representar cualidades propias de un fenómeno estudiado, y la modelación, la cual se enmarca en una serie de escenarios donde los estudiantes construyen conocimiento matemático, lo validan y lo generalizan de formas muy particulares.

Las consideraciones anteriores y otras reflexiones alrededor de la enseñanza y el aprendizaje de la trigonometría, ofrecieron insumos para plantear la siguiente pregunta de investigación: ¿Cómo a través de la modelación matemática los estudiantes producen algunos aspectos conceptuales asociados a la función trigonométrica seno? 


\section{Referentes teóricos}

Durante la última década son varios los investigadores que han aportado desde sus trabajos a la enseñanza de la matemática teniendo como estrategia didáctica la modelación (Burkhardt, 2006; Biembengut y Hein, 2004; Trigueros, 2009; VillaOchoa, 2007; Kaiser y Schwarz, 2010). Según la literatura internacional, la modelación permite establecer conexiones entre el "mundo real" y algunos conceptos matemáticos que se trabajan en el aula, no desde la intención de llenar de historia o de información un preámbulo dirigido al desarrollo de una clase; sino, con el propósito de generar conocimiento matemático desde el estudio en colectivo de un problema "ligado" a la "realidad" del estudiante. Dichos nexos son el resultado de un cambio en la forma como los docentes desarrollan sus clases de matemáticas trascendiendo la enseñanza de una matemática descrita en mallas curriculares; y optando por un proceso en el cual, el estudiante tenga otro rol que le permita ser partícipe de la construcción de conocimiento desde la experimentación, el análisis, la toma de decisiones y la estructuración de modelos matemáticos, entre otros. En este sentido, Trigueros (2009) apunta que "en la mayor parte de los acercamientos a la modelación se intenta, más bien, aprovechar las ideas que surgen de los estudiantes para introducir conceptos importantes de la matemática" (p. 80). Este tipo de consideraciones impone a los docentes una serie de necesidades y una gama más amplia de estrategias de enseñanza que favorezcan los procesos de aprendizaje.

En este ámbito, la modelación no es un proceso sencillo y al respecto, Burkhardt (2006) resalta la necesidad de usar la tecnología para tal fin, pues al parecer, las bondades que ésta ofrece, proporcionan un apoyo invaluable al establecimiento de estructuras de análisis, la variación producida por diferentes tipos de datos y la disposición de múltiples rutas de comprobación de los resultados. Desde esta mirada, en el trabajo se retomó, el constructo teórico Humans-with-media, desarrollado por Borba y Villarreal (2005); en el cual, la tecnología tiene un papel primordial en relación con el uso de experimentos en matemáticas y en la educación matemática; razón por la cual las matemáticas experimentales se han abierto campo impulsadas por el impacto de los computadores, además de las potencialidades de las calculadoras gráficas y diferentes interfaces que vinculados a ellos, pueden tomarse en conjunto como una unidad (humanoscon-los medios) reorganizante del pensamiento y de la forma misma como se produce conocimiento.

En el marco de uso de la tecnología en el aula, otro referente que se tomó en cuenta para este trabajo es el desarrollado por los investigadores Suárez y Cordero (2010), quienes hablan de la estructura propia que posee la modelación, constituida por un sistema dinámico, y colocan la simulación como un medio que permite el desarrollo del razonamiento y la argumentación. En esta perspectiva formulan un marco de referencia en la que convergen la modelación y la graficación para la construcción de nociones de cambio y de variación; la cual se fundamenta en tres premisas: 1) La graficación antecede a la función, ya que ésta permite la construcción de ideas de variación. (Funcionalidad-1). 2) La gráfica es argumentativa, ya que pasa a ser un elemento central de explicaciones y ello permite la construcción de argumentos. (Funcionalidad-2). 3) El uso de las gráficas permite encontrar generalizaciones que aportan al proceso de modelación; permite la cuantificación del movimiento y ciertas características asociadas con éste. (Funcionalidad-3). Algunos elementos de estos referentes, sirvieron de inspiración para el diseño de las simulaciones propuestas en la investigación y dieron pautas para analizar los resultados encontrados en la misma.

\section{Metodología}

Desde hace varios años, estudios realizados con metodologías cualitativas han estado presentes en diversos trabajos de investigación; en éstos se observa en términos de Quecedo y Castaño (2002), la producción de datos descriptivos, en relación con las palabras (habladas o escritas) de las personas involucradas en la investigación y las conductas observables. Los vínculos establecidos entre estudiantes, conocimiento e investigador, permitieron 
crear condiciones de validación y verificación de algunas consideraciones que emergieron en el desarrollo de la investigación, las cuales sirvieron de puente entre una apreciación propia de la realidad y un concepto matemático desarrollado desde la misma.

Una mirada a la pregunta de investigación permitió indagar acerca del proceso de modelación en educación matemática, su desarrollo, ventajas, limitaciones y la forma en que puede llevarse a cabo en el aula, motivo por el cual, se eligió el estudio de casos, como método de investigación puesto que en palabras de Yin (2009); las preguntas que se enfocan en el "cómo" o el "por qué" de un fenómeno social son especialmente un indicador para optar por dicho método para la investigación. En esta dirección se construyó una proposición (orientación) que dirigió la atención a situaciones particulares de interés para la investigación; y se enmarcó en: Observar la forma en que los estudiantes en unidad con los medios diseñados para la investigación, exteriorizan, por medio de representaciones, verbalizaciones, observaciones, o gesticulaciones, las interpretaciones que subyacen de la experimentación y simulación en escenarios dinámicos dotados de relaciones entre la medición y el movimiento de un reloj con elementos gráficos asociados a la función trigonométrica seno.

Los instrumentos que permitieron la obtención de información tuvieron sus bases en la observación participante, documentos y entrevistas; de ahí que todos los datos encontrados se analizaron a la luz de la relación entre la pregunta de investigación, el referente teórico y la información recolectada en el trabajo de campo.

\section{Un primer acercamiento a la trigonometría}

Los estudiantes vinculados a la investigación, trabajaron en parejas con simulaciones desarrolladas en el software Modellus 4. presentaron descripciones cualitativas, en las cuales prevalecieron las relaciones entre longitud de un vector, o el desplazamientos horizontal en la oscilación del péndulo, y el tipo de gráficos que ésto producía. Apoyados en sus observaciones, identificaron patrones que permitieron refinar el concepto de amplitud y periodo de la función trigonométrica vinculada a la simulación. Posteriormente los estudiantes construyeron las gráficas asociadas al movimiento de dos cuerpos, que se movían horizontalmente entre dos puntos, uno de ellos siguiendo la función seno, y el otro siguiendo una función "diente de sierra."

\section{Conclusiones}

El trabajo con simulaciones permitió que los estudiantes encontraran relaciones entre magnitudes, generalizaciones de la amplitud y elementos característicos del periodo de algunas funciones; además, permitieron encontrar evidencia de cómo ellos construyen elementos conceptuales relacionadas con la función trigonométrica seno. Estas relaciones halladas a partir de las gráficas, mostraron como surgen en paralelo a esta situación; una serie de argumentos que reconstruyeron las percepciones iniciales de los estudiantes y les ayuda a realizar transferencias cuando abordan otro tipo de situaciones diferentes, que generan la necesidad de refinar cada vez más sus conjeturas.

En resonancia con varios de los planteamientos de Suárez y Cordero (2008); Borba y Villarreal (2005), los estudiantes vinculados a la investigación en interacción con sus compañeros, medios y profesor, construyeron conocimiento matemático vinculado a unas situaciones de movimiento, presentes en interfaces donde se desarrolló el proceso de modelación.

Agradecimientos a la Universidad de Medellín, ya que en el marco de la Maestría en Educación Matemática se desarrolló este estudio con la asesoría de Jhony Alexander Villa-Ochoa; también a los miembros de la Red Colombiana de Modelación en Educación Matemática (RECOMEM) quienes ofrecieron sugerencias para la cualficaciòn de este documento. 


\section{Referencias}

Kaiser, G., \& Schwarz, B. (2010). Authentic Modelling Problems in Mathematics Education-Examples and Experiences. Journal für Mathematik-Didaktik, 31(1), 52-76.

Biembengut, M., \& Hein, N. (2004). Modelación matemática y los desafios para enseñar matemática. Educación matemática, 16 (2),105-125.

Borba, M. C., \& Villarreal, M. E. (2005). Humanswith-Media and the Reorganization of Mathematical Thinking. New York: Springer.

Burkhardt, H. (2006). Modelling in mathematics classrooms: reflections on past developments and the future. ZDM, 38 (2), 178-195.

Kaiser, G., \& Sriraman, B. (2006). A global survey of international perspectives on modelling in mathematics education. ZDM, 38(3), 302-310.

MEN, M. (2006). Estandares Básicos de Competencias en Matemáticas. Bogotá: Cooperativa Editorial Magisterio.

Mora, M. F., Nieto, E. X., Polanía, D. L., Romero, M. L., \& González, M. J. (2012). Razones trigonométricas vistas a través de múltiples lentes. En P. Gómez, Diseño, implementación y evaluación de unidades didácticas matemáticas en MAD 1 (págs. 261-341). Bogotá: Universidad de los Andes.

Quecedo, L., \& Castaño, c. (2002). Introducción a la metodología de investigación cualitativa. Revista de Psicodidáctica, 14, 5-40.

Suárez, L., \& Cordero, F. (diciembre de 2010). Modelación-graficación, una categoría para la matemática escolar. Resultados de un estudio socioepistemológico. Revista latinoamericana de investigación en educación matemática, 13 (4-II), 319-333.

Trigueros, M. (2009). El uso de la modelación en la enseñanza de las matemáticas. Innovación Educativa, 9 (46),75-87.

Villa-Ochoa, J. (2007). La modelación como proceso en el aula de matemáticas, un marco de referencia y un ejemplo. Tecno Lógicas, 19, 63-85.

Yin, R. K. (2009). Case study research, Design and methods. Thounsand Oaks, California: Sage Publications, Inc. 